Monatsschr Kinderheilkd 2020 · 168:739-743 https://doi.org/10.1007/s00112-020-00989-7 Online publiziert: 14 . Juli 2020

(c) Springer Medizin Verlag GmbH, ein Teil von Springer Nature 2020

\section{Redaktion}

A. Borkhardt, Düsseldorf

S. Wirth, Wuppertal

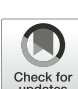

\section{B. Lange ${ }^{1,3} \cdot$ T. Tenenbaum ${ }^{2} \cdot$ L. M. Wessel ${ }^{3}$}

'Stabsstelle Krankenhaushygiene, Medizinische Fakultät Mannheim, Universität Heidelberg, Universitätsmedizin Mannheim, Mannheim, Deutschland

${ }^{2}$ Klinik für Kinder- und Jugendmedizin, Medizinische Fakultät Mannheim, Universität Heidelberg, Universitätsmedizin Mannheim, Mannheim, Deutschland

${ }^{3}$ Kinderchirurgische Klinik, Medizinische Fakultät Mannheim, Universität Heidelberg, Universitätsmedizin Mannheim, Mannheim, Deutschland

\title{
COVID-19-Pandemie: Management kinderchirurgischer Patienten
}

\section{Stellungnahme der Deutschen Gesellschaft für Kinderchirurgie (DGKCH), der Deutschen Gesellschaft für Kinder- und Jugendmedizin (DGKJ) und der Deutschen Gesellschaft für pädiatrische Infektiologie (DGPI)}

\section{Einleitung}

Die weltweite Ausbreitung des Erregers SARS-CoV-2 (neuartiges Coronavirus), welches als Ursache der „Coronavirus Disease 2019“ (COVID-19) identifiziert werden konnte, wurde am 11.03.2020 von der WHO zur Pandemie erklärt. Um bei steigenden Fallzahlen auch in Deutschland die stationäre Versorgung COVID19-positiver Patienten gewährleisten zu können, wurde am 13.03.2020 gemäß Infektionsschutzgesetz (IfSG) \$32 die „Verordnung zur Bekämpfung des Coronavirus" erlassen. In dieser sind elektive Aufnahmen, Eingriffe oder Operationen auf unbestimmte Zeit zu verschieben und das Besuchsrecht in den Kliniken einzuschränken [8].

Mit Stand vom 10.04.2020 wurde vom Robert Koch-Institut (RKI) anhand der Meldepflicht gemäß IfSG über 113.525 positive COVID-19-Fälle in Deutschland berichtet $[23,24]$. Von diesen waren 2228 Kinder in der Altersgruppe 5 bis 14 und 882 in der Altersgruppe 0 bis 4 Lebensjahre zu finden. Publikationen aus China, Italien, Spanien und den USA bestätigen die Beobachtung eines nur geringeren
Erkrankungsrisikos mit einer Inzidenz von $1-5 \%$ und das Ausbleiben schwerer Krankheitsverläufe bei Fehlen von Risikofaktoren im Kindesalter [11, 15, 16, 29]. Ein Todesfall wurde bei Kindern und Jugendlichen in Deutschland im Rahmen einer Umfrage der Deutschen Gesellschaft für Pädiatrische Infektiologie (DGPI Survey) registriert [3].

Im Umgang mit COVID-19 stehen in den Kliniken infektionshygienisch primäre (Basishygiene in Form von Händedesinfektion, Isolierung/Kohortierung, persönlicher Schutzausrüstung [PSA] u. a.) und sekundäre (Surveillance-)Präventionsmaßnahmen im Vordergrund. Im Sinne des ressourcensparenden Umgangs mit Test-Kits wurde zu Beginn gemäß RKI-Vorgabe nicht jeder stationär aufzunehmende Patient, sondern risikoadaptiert bei positiver Anamnese und typischen Symptomen wie Fieber, Husten, grippeähnlichen Symptomen und Störung des Geruchs-/Geschmackssinns (begründeter Verdachtsfall) auf SARSCoV-2 getestet.

Infolge der zunehmenden Testkapazität, sinkender Fallzahlen und Lockerung des Shutdowns definierte das RKI am 07.05.2020 den Beginn einer neuen Phase der COVID-19-Pandemie. So waren am 16.05.2020 in Deutschland nur noch ca. 15.000 akut Erkrankte/Infizierte registriert, von denen bundesweit 400 das Kindesalter betrafen. Am 19.05.2020 wurde vom Bundestag mit Zustimmung des Bundesrates das „Zweite Gesetz zum Schutz der Bevölkerung bei einer epidemischen Lage von nationaler Tragweite " beschlossen. In diesem Gesetz wird der persönliche Anspruch auf Testungen für den Nachweis einer SARS-CoV2-Infektion festgeschrieben. In der am 03.06.2020 publizierten AWMF-S1-Leitlinie „Interdisziplinär abgestimmte Empfehlungen zum Personal- und Patientenschutz bei Durchführung planbarer Eingriffe zur Zeit der SARS-CoV-2-Pandemie " [13] wird für alle Patienten die Empfehlung zur Durchführung eines PCRAbstrichs maximal drei Tage vor einer elektiven Operation ausgesprochen.

Wir empfehlen daher wie in der AWMF-Leitlinie „in Abhängigkeit der regionalen epidemiologischen Situation für alle Patienten ohne mögliche $\mathrm{CO}$ VID-19-assoziierte Symptome vor einem elektiven bzw. auch kurzfristig planbaren 
ambulanten und stationären Eingriff die Durchführung einer präoperativen PCRAbstrich-Testung. Auf die Abstrichtestung kann verzichtet werden, falls der regionale öffentliche Gesundheitsdienst (das für die Region zuständige $\mathrm{Ge}$ sundheitsamt) aufgrund sehr niedriger Neuinfektionszahlen eine solche globale Testung für nicht erforderlich hält bzw. die jeweilige regionale epidemiologische Situation es zulässt." Dieses Vorgehen steht im Einklang mit der ,Verordnung zum Anspruch auf bestimmte Testungen für den Nachweis des Vorliegens einer Infektion mit SARS-CoV-2“ des Bundesministeriums für Gesundheit vom 09.06.2020, wonach unter Berücksichtigung der jeweiligen epidemiologischen Lage PCR-Abstriche vor elektiven Eingriffen durchgeführt werden können, aber nicht müssen.

Unter Berücksichtigung dieser Empfehlungen sollten zukünftig Patienten, die wegen einer chirurgischen Indikation ambulant oder stationär aufgenommen werden, einer SARS-CoV-2-Diagnostik zugeführt werden. Diese Ausweitung von Tests bezieht insbesondere auch asymptomatische Kinder und deren Begleitperson ein, um in Abhängigkeit von der epidemiologischen Situation eine eventuelle Dunkelziffer und damit mögliche Infektionsgefahr aufspüren zu können. Diese ausgedehnte Teststrategie kann den Schutz des Personals stärken, die Verbreitung des Virus und das Risiko eines Ausbruchgeschehens reduzieren sowie die Morbidität der Patienten senken. Kritisch müssen der aktuell noch hohe Kostenfaktor der SARS-CoV-2Testung, die Möglichkeit falsch-positiver bzw. falsch-negativer Testergebnisse, der logistische Aufwand der Testung vor der stationären Aufnahme und die potenziell unangenehme Abstrichprozedur gegenübergestellt werden.

Es zeigt sich hier eine Ähnlichkeit mit früheren Coronaviruserkrankungen wie SARS und MERS. Auch bei diesen Epidemien waren Kinder deutlich weniger häufig betroffen und erkrankten weniger schwer im Gegensatz zu Erwachsenen. Obwohl innerhalb der Familien eine Ansteckung durchaus auftrat, waren Kinder in ca. zwei Drittel der Fälle nur sehr leicht erkrankt oder gar asymptomatisch $[1,26]$.

Bis Anfang Mai 2020 kam trotz der niedrigen Infektionszahlen im Kindesalter infolge innerklinischer personeller Umverteilung und mit dem Ziel der Reduktion von Kontakten auch in kinderchirurgischen Kliniken neben dem Absetzen aller elektiven Operationen und dem eingeschränkten Besuchsrecht für Angehörige auch die ambulante Tätigkeit in den Spezialsprechstunden nahezu vollständig zum Erliegen [7]. Infolgedessen war es niedergelassenen Kinderärzten nicht möglich, Patienten in den Spezialambulanzen vorzustellen. Ursächlich waren beibekannten knappen Personalressourcen auch die aufgrund der Pandemie veranlasste Separierung des Personals innerhalb der Klinik sowie die Verlagerung des Fachpersonals in potenzielle COVID-19-Bereiche. Es drohte die Gefahr, dass Patienten daher häufig verzögert oder im Einzelfall gar zu spät vorgestellt wurden. Einzelberichte zeigen, dass eine verzögerte Vorstellung in der Klinik zu komplizierten Verläufen führen kann [14].

Hinzu kommt weiterhin die Angst der Eltern, in Zeiten der COVID-19Pandemie bei einem Besuch oder Aufenthalt in der Klinik einem erhöhten Infektionsrisiko ausgesetzt zu sein. Dies gilt vor allem für Kinder mit chronischen Erkrankungen und Immunsuppression, obwohl die Gefährdung im Vergleich $\mathrm{zu}$ Erwachsenen deutlich geringer $\mathrm{zu}$ sein scheint [11]. Unterstützt durch die zuständige Krankenhaushygiene müssen durch regelmäßige Surveillance nosokomiale SARS-CoV-2-Infektionen in den Kliniken rechtzeitig erkannt bzw. vor allem vermieden werden.

Auch in Kinderzentren muss jedoch vor allem in Zeiten einer Pandemie die klare Trennung infektiöser Patienten sowohl in der ambulanten Versorgung als auch stationären Behandlung ein elementarer Bestandteil der gelebten Basishygiene sein. Organisatorisch gehören dazu bereits für Verdachtsfälle die getrennte Wegeführung und räumliche Separierung in einem eigenen Behandlungsraum in der Notaufnahme.

\section{Auswirkungen auf die Kinderchirurgie in der ersten Phase der Pandemie}

Unabhängig von COVID-19 werden auch im Kindesalter chirurgische Eingriffe in Notfalloperationen, dringliche und elektive Eingriffe eingeteilt. Operationen im Fachgebiet Kinderchirurgie sind jedoch zusätzlich durch ihre Abhängigkeit vom Reifegrad und Entwicklungsstand der Kinder gekennzeichnet.

Auf den ersten Blick lässt in Kinderzentren mit eigener pädiatrischer Intensivstation die äußerst geringe Zahl schwer kranker, intensivpflichtiger COVID-19Fälle im Vergleich zu Erwachsenen vermeintlich mehr Handlungsspielraum für die postoperative Versorgung zu. Insgesamt muss jedoch berücksichtigt werden, dass auch in kinderchirurgischen Kliniken die eingeschränkte Operationskapazität durch Personalverlagerung aus dem Operations- in den Intensivbereich (Anästhesie) und die Verlagerung von Intensivpersonal aus dem Kinder- in den Erwachsenbereich (z. B. ECMO-Expertise) zu einer Einschränkung der Operationskapazität führen kann. Die Benennung zweier voneinander getrennter Behandlungsteams soll innerhalb einer Klinik sicherstellen, dass im Fall einer COVID19-Erkrankung das zweite Team mit derselben Fachexpertise zeitnah zur Verfügung steht [19]. Kleinere Kliniken sollten durch eine gezielte Personaleinsatzplanung unnötige Personalwechsel zwischen den Bereichen vermeiden. Die COVID-19-Pandemie führt daher auch im pädiatrischen Bereich zu Versorgungsengpässen durch Absetzen von Elektivoperationen und reduzierte Intensivkapazitäten.

Zahlreiche Notfalleingriffe müssen trotz diskutierter Therapiealternativen auch während einer Pandemie in der Kinderchirurgie durchgeführt werden. Im Bereich der Traumatologie zählen dazu unter anderem die Versorgung von Frakturen und Polytraumata, thermischen Verletzungen und endoskopische Fremdkörperentfernungen (Ösophagus, Bronchialsystem). Darüber hinaus Eingriffe in der Viszeralchirurgie (Ileus, Volvulus, nekrotisierende Enterokolitis), Gynäkologie (Ovarialtorsion) und 
Urologie (posteriore Urethralklappe, Urosepsis infolge Obstruktion, Hodentorsion, symptomatische Phimose/ Paraphimose [22]). COVID-19-positive Patienten mit Appendizitis können nach ausführlicher Aufklärung der Eltern im Einzelfall auch primär konservativ behandelt werden. Alternativ bietet sich bei perforierter Appendizitis die Abszessdrainage mit Intervallappendektomie an [21]. Differenzialdiagnostisch muss bei akuter gastrointestinaler Symptomatik mit Fieber und Zeichen der Hyperinflammation an ein COVID-19-assoziiertes „multisystem inflammatory syndrome in children" (MIS-C) mit Multiorganversagen und Schock gedacht werden [28]. Ergänzend kommen bei Neugeborenen die operative Versorgung angeborener Fehlbildungen (wie kongenitale Zwerchfellhernie, sämtliche Atresien, Bauchwanddefekte) und bei Säuglingen irreponible oder eingeklemmte Hernien (Leistenhernie, Nabelhernie) hinzu.

$\mathrm{Zu}$ den dringlichen Eingriffen, die innerhalb von 30 Tagen durchgeführt werden sollten, zählen unter anderem die operative Anlage eines getunnelten, zentralvenösen Kathetersystems zur Chemotherapie oder parenteralen Ernährung bei Kurzdarmsyndrom. Onkologische Behandlungen (Nephroblastom, Neuroblastom) sollten weiterhin priorisiert, interdisziplinär anhand standardisierter Therapieprotokolle geplant und aufgrund der in den letzten Jahren verbesserten Ergebnisse weitgehend zeitgerecht durchgeführt werden. Demgegenüber muss bei allgemein eingeschränkten Ressourcen in der Pandemie der Zeitpunkt der geplanten Korrektur eines Hodenhochstands mit den Eltern kritisch besprochen werden. Metallentfernungen bei Implantaten nahe bzw. mit Kreuzung der Wachstumsfuge sollten ebenfalls nicht unnötig verschoben werden. Nachteile in Form von Fertilitätseinschränkung oder Wachstumsstörung müssen durch den korrekten Operationszeitpunkt minimiert, die in der Pandemie jedoch eingeschränkten Personal-, Betten- und OP-Kapazitäten ebenfalls berücksichtigt werden.

\section{Infektiologische Aspekte}

Für jeden Eingriff sollte individuell berücksichtigt werden, dass bei gleichzeitiger viraler Infektion des Respirationstraktes die postoperative Rekonvaleszenz verkompliziert und verlängert werden kann [30]. Bislang gibt es nur wenige Publikationen, die den postoperativen klinischen Verlauf nach stattgehabter Operation und Nachweis einer akuten Infektion durch respiratorische Viren dokumentiert haben. In einer chinesischen Studie mit 1629 Kindern, die sich einer Operation unterziehen mussten, konnte bei 171 Kindern mindestens ein viraler respiratorischer Erreger gefunden werden. Wichtige Erreger wie RSV (9,9\%) und Parainfluenzavirus 3 $(24,5 \%)$ konnten dabei mit hoher Viruslast nachgewiesen werden, welches als Indiz für eine akute Infektion zu sehen ist. Initial waren diese Kinder meist wenig symptomatisch, verschlechterten sich dann aber mit dem klinischen Bild einer Pneumonie [19]. In einer großen australischen Studie mit kardiochirurgisch behandelten Kindern fand sich bei 73 von $1723(4,2 \%)$ der Nachweis von respiratorischen Viren. Der Verlauf war bei diesen Kindern durch einen längeren Aufenthalt auf der Intensivstation und längere Intubationsdauer kompliziert worden [18]. In einer kleineren amerikanischen Studie kam man zu einem ähnlichen Ergebnis [9]. Darin war der Aufenthalt auf der Intensivstation sogar um das Dreifache verlängert. Bei einer kleinen Patientengruppe von 12 Kindern mit Lebertransplantation konnte eine erhöhte Komplikationsrate von arteriellen Thrombosen bei Nachweis von respiratorischen Viren demonstriert werden [12]. Bisherige anästhesiologische Empfehlungen sahen vor, Kindern mit moderaten und schweren Erkältungssymptomen vom OPProgramm abzusetzen und nach zwei Wochen wieder einzubestellen. Bei milden Symptomen wird das abhängig vom Eingriff nicht ganz so kritisch gesehen [4]. Da bei Kindern mit SARS-CoV-2Infektion die Klinik meist milde oder gar asymptomatisch verläuft, muss die Dringlichkeit der Indikation zu einer
Operation auch bei milder Symptomatik kritisch überprüft werden.

\section{Hygienische Aspekte}

Auch bei asymptomatischen Kindern sollte aus Gründen des Mitarbeiterschutzes maximal $72 \mathrm{~h}$ präoperativ ein SARS-CoV-2-Abstrich erfolgen. Wünschenswert wäre allerdings eine Testung innerhalb $24 \mathrm{~h}$ oder gar mittels „Pointof-care"-Schnelltest innerhalb weniger Minuten, sodass die Patientenlogistik optimiert wäre. Aktuell bleibt labortechnisch bedingt weiterhin eine gewisse Unklarheit. Die Eltern müssen nach Abnahme des Abstrichs bis zum vereinbarten Operationstermin das Kind gegenüber Nichtangehörigen des eigenen Haushalts abschirmen. Das Ergebnis des Abstrichs bestimmt den Zeitpunkt der elektiven Aufnahme und im Einzelfall den Zeitpunkt des chirurgischen Eingriffs.

Elektive Patienten mit prophylaktischem Screening werden auf die kinderchirurgische Normalstation nur bei negativem Testergebnis aufgenommen. Sollte ein notfallmäßig aufgenommener Patient zufällig SARS-CoV-2-positiv sein, muss eine entsprechende Verfolgung der Kontaktpersonen bzw. des Personals erfolgen und eine Umgebungsuntersuchung eingeleitet werden. Hier sind dann entsprechend die Krankenhaushygiene, der Betriebsarzt und die lokale Gesundheitsbehörde zu involvieren.

Die notfallmäßige Aufnahme von Kindern mit chirurgischem Krankheitsbild und zusätzlich gesicherter SARSCoV-2-Infektion sowie von Kindern mit COVID-19-spezifischen Symptomen ohne Testergebnis oder nach ungeschütztem Kontakt zu einer COVID19-positiven Person sollte in einer separaten Station oder in einem separaten Stationsbereich bis zum Vorliegen des Testergebnisses erfolgen. Bei negativem Testergebnis sollte bei weiterhin zusätzlich bestehenden COVID-19-typischen Symptomen eine zweite Testung (z. B. Sputum) durchgeführt werden. Bei ebenfalls negativem zweiten Testergebnis kann dann die Verlegung auf die Normalstation erfolgen. 
In der Indexstation werden die $\mathrm{Pa}$ tienten nach „Verdachtsfall“ und „gesichertem Fall" in einem Einzelzimmer mit Nasszelle untergebracht. Bei hohem Patientenaufkommen können sie diagnosebezogen auch kohortiert werden. Angesichts der Tröpfcheninfektion durch SARS-CoV-2 sollte im optimalen Fall ein zusätzlicher Vorraum vorhanden sein, in dem die persönliche Schutzausrüstung (PSA) angelegt wird. Die PSA besteht standardmäßig aus Schutzbrille, FFP2Maske, Schutzkittel und Schutzhandschuhen. Im Rahmen des ressourcensparenden Umgangs mit PSA können die Einmalprodukte Schutzbrille und FFP2Maske mitarbeiterbezogen mehrfach verwendet bzw. die Schutzbrille auch maschinell aufbereitet werden. Schutzkittel und Schutzhandschuhe werden nach Gebrauch im Zimmer entsorgt. Die Versorgung durch speziell im Umgang mit COVID-19-positiven Patienten geschultes Personal wird dringend empfohlen.

\section{Organisatorische Voraus- setzungen}

Die Etablierung eines speziell für COVID-19-positive Patienten vorgesehenen OP-Bereichs ergibt infektionshygienische Vorteile [27]. Dies betrifft sowohl das Training des Personals, die Ausstattung des OP-Saals als auch die RaumluftTechnische(RLT)-Anlage [20]. Die RLTAnlage wirkt für Patienten und alle Mitarbeiter jedoch nur dann infektionspräventiv, wenn die Türen des OPSaals dauerhaft geschlossen sind [10]. In diesem OP-Saal sollte sich aus infektionspräventiven Gründen die Zahl der anwesenden Mitarbeiter auf das absolut notwendige Minimum beschränken und gemäß RKI die Personalfluktuation und das Sprechen auf ein Mindestmaß reduzieren.

Für die Mitarbeiter der Anästhesie gilt sowohl bei hohem Verdacht auf COVID19 und noch fehlendem Ergebnis des SARS-CoV-2-Abstrichs als auch bei gesicherter COVID-19-Diagnose für den gesamten operativen Eingriff, einschließlich Intubation und Extubation, die Anlage von Schutzbrille und FFP3-Maske. Das Team aus Kinderchirurgie mit Assis- tenz und instrumentierender Pflegekraft betritt den kinderchirurgischen OP erst nach Intubation des Patienten und trägt bei Verdacht auf COVID-19 oder gesicherter COVID-19-Diagnose für die Zeit des Eingriffs Schutzbrille und FFP2-Maske. Die Extubation des Patienten erfolgt erst, wenn das OP-Team den OP-Saal verlassen hat.

Unabhängig vom SARS-CoV-2-Status des Patienten wird vom Anästhesisten präoperativ die von der Klinik festgelegte perioperative Antibiotikaprophylaxe (PAP) appliziert. Im Team-Time-Out vor Hautschnitt sollte in Zeiten der COVID-19-Pandemie zusätzlich die korrekte PSA aller im OP-Saal beteiligten Personen überprüft werden.

Für abdominalchirurgische Eingriffe sollte präoperativ eine Nutzen-RisikoBewertung hinsichtlich konventioneller vs. laparoskopischer Technik erfolgen. Die Vorteile der minimal-invasiven Operationstechnik wurden auch für Kinderund Jugendliche unter den Aspekten der besseren Übersicht, geringeren Infektionsraten für den Chirurgen, kürzeren Verweildauer, des geringeren postoperativen Schmerzes mit geringerer postoperativer Infektionsrate aufseiten des Patienten publiziert. Demgegenüber wird die mögliche Aufnahme von SARSCoV-2-Partikeln als infektiöses Aerosol über den chirurgischen Rauch und das Pneumoperitoneum diskutiert [2, 5, 22]. Gemäß aktuellem Stand der Wissenschaft kann eine solche Übertragung nicht vollständig ausgeschlossen werden. Allerdings bleibt unsicher, ob die nachgewiesenen Viruspartikel im Aerosol tatsächlich noch infektiös sind. Im Sinne des allgemeinen Infektionsschutzes sollte angesichts der COVID-19-Pandemie von Kinderchirurgen und Industrie diese Problematik erneut aufgegriffen werden, um generell das mögliche Infektionsrisiko für das OP-Team durch das Pneumoperitoneum zu reduzieren. Dazu gehören die standardisierte Begrenzung des intraabdominellen Druckniveaus, der Gebrauch einer Absaugung mit Rauchgasfilter, die Anwendung dichter Trokare sowie die Nutzung eines Partikelfilters beim Gasauslass [6, 17, 19, 22, 25, 31]. Das unkontrollierte und ungeschützte Ablassen des Pneu- moperitoneums über den Trokar zum Bergen von Gewebe (z.B. Appendix) oder am Ende des operativen Eingriffs sollte unterbleiben, oder alle weiteren Mitarbeiter müssen währenddessen den OP-Saal verlassen. Nach derzeitigem Wissensstand sollte bei Fehlen der oben genannten Schutzausrüstung und mangelhafter technischer Ausstattung von der laparoskopischen OP-Technik Abstand genommen werden.

\section{Auswirkungen auf die Kinderchirurgie in der zweiten Phase der Pandemie}

Um auch in den kinderchirurgischen Kliniken die Umsetzung des vom Bundesministerium für Gesundheit geforderten "neuen Klinikalltags“ bedarfsgerecht umsetzten zu können, ist neben der Anpassung infektionspräventiver und organisatorischer Maßnahmen die strikte Aufrechterhaltung der bereits in der ersten Phase der Pandemie etablierten Basishygiene erforderlich.

Zukünftig wird für alle stationären Patienten und deren Begleitperson routinemäßig vor bzw. unmittelbar nach Aufnahme einen SARS-CoV-2-Abstrich empfohlen. Darüber hinaus sollten die Begleitpersonen und Besucher einen Selbstauskunftsbogen zum eigenen Gesundheitsstatus und eventuell stattgehabter Kontakte mit COVID-19-positiven Personen ausfüllen. Das Tragen eines Mund-Nasen-Schutzes ist sowohl für Mitarbeiter als auch Patienten und deren Begleitperson bei Bewegung innerhalb der Klinik obligat. Die räumliche und organisatorische Trennung infektiöser von nichtinfektiösen Patienten gilt sowohl für die Erstversorgung/Aufnahme, die ambulante Diagnostik als auch die stationäre Versorgung. Bei Verdacht oder gesicherter SARS-CoV-2-Infektion muss weiterhin eine Indexstation („floating station“) oder ein organisatorisch getrennter Stationsbereich genutzt werden, die Kapazität kann jederzeit bedarfsadaptiert angepasst werden. Bei sorgfältiger Händehygiene, Respektierung eines Personenabstands von mindestens 1,5 m und einer guten Raumlüftung ist neben der Wiederöffnung der (Spezial-)Ambulanzen auch das Hoch- 
fahren der elektiven Operationstätigkeit möglich. Dabei muss jedoch in enger Zusammenarbeit mit der hauseigenen Krankenhaushygiene und den zuständigen Gesundheitsämtern die InfektionsSurveillance engmaschig analysiert und bei Bedarf an die Situation angepasst werden.

\section{Fazit für die Praxis}

\section{- Organisatorische Unterscheidung zwischen Notfalleingriffen, dringli- chen und elektiven Eingriffen in der Pandemie zwingend erforderlich. \\ - Empfehlung zur Durchführung eines präoperativen SARS-CoV-2-Abstrichs auch bei asymptomatischen Kindern. \\ - Zu jeder Zeit strikte Einhaltung der Basishygiene notwendig. \\ - Tragen eines Mund-Nasen-Schutzes aufseiten der Behandler, Patienten und Eltern. \\ - Bereitstellung von Schutzausrüstung (Schutzbrille, FFP2-Maske/FFP3-Mas- ke, Schutzkittel, Schutzhandschuhe) für das Behandlungsteam. \\ - Zur operativen Versorgung COVID- 19-positiver Patienten Etablierung eines separaten OP-Bereichs. \\ - Bei laparoskopischen Eingriffen Nut- zung von Absaugvorrichtungen für Rauchgas und das Pneumoperitone- um.}

\section{Korrespondenzadresse}

\section{Dr. B. Lange}

Stabsstelle Krankenhaushygiene, Medizinische Fakultät Mannheim, Universität Heidelberg, Universitätsmedizin Mannheim

Theodor-Kutzer-Ufer 1-3, 68167 Mannheim, Deutschland

bettina.lange@umm.de

\section{Einhaltung ethischer Richtlinien}

Interessenkonflikt. B. Lange, T. Tenenbaum und L.M. Wessel geben an, dass kein Interessenkonflikt besteht.

Für diesen Beitrag wurden von den Autoren keine Studien an Menschen oder Tieren durchgeführt. Für die aufgeführten Studien gelten die jeweils dort angegebenen ethischen Richtlinien.

\section{Literatur}

1. Alfaraj SH, Al-Tawfiq JA, Altuwaijri TA, Memish ZA (2019) Middle east respiratory syndrome coronavirus in pediatrics: a report of seven cases from Saudi Arabia. Front Med 13:126-130

2. Alp E, Bijl D, Bleichrodt RP, Handsson B, Voss A (2006) Surgical smoke and infection control. JHosp Infect 62:1-5

3. Armann JP, Simon A, Diffloth N, Doenhardt M, Hufnagel M, Trotter A, Scheider D, Hübner J, Berner R (2020) Hospital admission in children and adolescents with COVID-19. Early results from a national survey conducted by the German Society for Pediatric Infectious Diseases (DGPI). Dtsch Arztebl Int 117:373-374

4. Becke K (2012) Anesthesia in children with a cold. Curr Opin Anaesthesiol 25:333-339

5. Bigony L (2007) Risks associated with exposure to surgical smoke plume: a review of the literature. AORN J 86:1013-1020

6. Brown J (2020) Surgical decision making in the era of COVID-19. A new set of rules. J Minim Invasive Gynecol 27(4):785-786

7. Bundesministerium der Justiz und Verbraucherschutz (2020) Gesetz zum Schutz der Bevölkerung bei einer epidemischen Lage von nationaler Tragweite. Bundesgesetzblatt I(14):587

8. Bundesministerium für Justiz und Verbraucherschutz (2020) Verordnung über die Ausdehnung der Meldepflicht nach $\S 6$ und $\S 7$ des Infektionsschutzgesetzes. Infektionsschutzgesetz, 1. Febr. 2020

9. Delgado-Corcoran C, Witte MK, Ampofo K, Castillo R, Bodily S, Bratton SL (2014) The impact of human rhinovirus infection in pediatric patients undergoing heart surgery. Pediatr Cardiol 35(8):1387-1394

10. Deutsche Gesellschaft für Krankenhaushygiene (2015) Krankenhaushygienische Leitlinie für die Planung, Ausführung und den Betrieb von Raumlufttechnischen Anlagen in Räumen des Gesundheitswesens. Hyg Med 40:519-526

11. Hedrich C (2020) Covid-19-considerations for the paediatric rheumatologist. Clin Immunol 214:108420

12. Kohler JA, Munoz FM, Goss JA, Miloh TA (2017) Viral upper respiratory infection at pediatric liver transplantation is associated with hepatic artery thrombosis. Liver Transpl 11:1477-1481

13. (2020) Interdisziplinär abgestimmte Empfehlungen zum Personal- und Patientenschutz bei Durchführung planbarer Eingriffe zur Zeit der SARS-CoV2-Pandemie. https://www.awmf.org/uploads/ tx_szleitlinien/017-080l_S1_EmpfehlungenPersonal-Patientenschutz-Eingriffe-SARS-CoV2-Pandemie_2020-06_1.pdf.Zugegriffen:18.Juni 2020

14. Lazzerini M, Barbi E, Apicella A, Marchetti $F$ Cardinale F, Trobia G (2020) Delayed access or provision of care in Italy resulting from fear of COVID-19. Lancet Child Adolesc Health 4(5):e10-e11. https://doi.org/10.1016/S23524642(20)30108-5

15. Livingston $\mathrm{E}$, Bucher $\mathrm{K}$ (2020) Coronavirus disease 2019 (COVID-19) in Italy. JAMA. https://doi.org/10. 1001/jama.2020.4344

16. Ludvigsson JF (2020) Systematic review of COVID-19 in children show milder cases and a better prognosis than adults. Acta Paediatr 109(6):1088-1095

17. Morris SN, Fader AN, Milad MP, Dionisi H (2020) Understanding the "scope" of the problem: why laparoscopy is considered safe during the
COVID-10 pandemic. J Minim Invasive Gynecol 27(4):789-791

18. Moynihan K, Barlow A, Alphonso N, Anderson B, Johnson J, Nourse C, Schlebusch S, Karl TR, Schlapbach LJ (2017) Impact of viral respiratory pathogens on outcomes after pediatric cardiac surgery. Pediatr Crit Care Med 18(3):219-227

19. Nassar AH, Zern NK, Mclntryre L, Lynge D, Smith CA, Petersen RP, Horvath DK, WoodDE (2020) Emergency restructuring of a general surgery residenca program during the coronavirus disease 2019 pandemic: the University of Washington experience. JAMA Surg. https://doi.org/10.1001/ jamasurg. 2020

20. Peloso A, Moeckli B, Oldani G, Triponez F, Toso C (2020) Response for a European surgical department to the COVID-19 crisis. Swiss MedWkly 150:w20241

21. Polites SF, Azarow KS (2020) Perspectives on pediatric appendicitis and appendectomy during the severe acute respiratory syndrome coronavirus 2 pandemic. J Laparoendosc Adv Surg Tech 30(4):356-357

22. Quaedackers JSLT, Stein R, Bhatt N, Dogan HS, Hoen L, Nijman RJM, Radmayr C, Silay MS, Tekgul S, Bogaert G (2020) Clinical and surgical consequences of the COVID-19 pandemic for patients with pediatric urological problems. Statement of the EAU guidelines panel for paediatric urology. J Pediatr Urol 16(3):284-287

23. Robert Koch-Institut (2020) COVID-19 Dashboard. https://corona.rki.de. Zugegriffen: 18. Juni 2020

24. Schilling J, Diercke M, Altmann D, Haas W, Buda S (2020) Vorläufige Bewertung der Krankheitsschwere von COVID-19 in Deutschland basierend auf übermittelten Fällen gemäß Infektionsschutzgesetz. Epidemiol Bull 17:3-9

25. Society of American Gastrointestinal and Endoscopic Surgeons (2020) Resources for smoke and gas evacuation during open, laparoscopic and endoscopic procedures (Webmaster)

26. Su L, Ma X, Yu JH, Su L, Ma X, Yu H, Zhang Z, Bian P, Han Y, Sun J, Liu Y, Yang C, Geng J, Zhang Z, Gai Z (2020) The different clinical characteristics of corona virus disease cases between children and their families in China-The character of children with COVID-19. Emerg Microbes Infect 9(1):707-713

27. Wong J, Goh QT, Tan Z, Lie SA, Tay YC, Ng SY, Soh CR (2020) Preparing for a COVID-19 pandemic: a review of operating room outbreak response measures in a large tertiary hospital in Singapore. Can J Anaesth 67(6):732-745

28. World Health Organization (2020) Multisystem inflammatory syndrome in children and adolescents temporally related to COVID-19 (Scientific Brief)

29. Wu Z, McGoogan J (2020) Characteristics of and important lessons from the Coronavirus disease 2019 (COVID-19) outbreak in China: summary of a report of 72314 cases form the Chinese Center for Disease Control and Prevention. JAMA. https://doi. org/10.1001/jama.2020.2648

30. Zhang D, Lou X, Yan H, Pan J, Mao H, Tang H, Shu Y, Zhao Y, Liu L, Li J, Chen D, Zhang Y, Ma X (2019) Respiratory virus associated with surgery in children patients. Respir Res 20(1):126

31. Zheng MH, Boni L, Fingerhut A (2020) Minimally invasive surgery and the novel coronavirus outbreak: lessons learned in China and Italy. Ann Surg 272(1):e5-e6. https://doi.org/10.1097/SLA. 00000000000003924 